tés et les améliorations. Elle devra enfin travailler dans les campagnes à faire connaître les avantages de l'électricité aux communes et à les inciter à s'en servir.

Ici, il est bon de remarquer que le moyen le plus sûr pour l'Etat de faciliter l'utilisation des chutes d'eau, serait sans aucun doute d'accorder des prêts d'Etat pour les établissements d'énergie hydro-électrique d'utilité publique et pour les lignes de transport de l'énergie. Ces prêts ont certainement autant de raison d'être que les prêts consentıs aux chemins de fer privés qui sont coutumiers en Suède. L'Etat aurait ainsi en même temps la possibilité d'exercer un contrôle sur l'économie des entreprises hydro-électriques, par exemple par le moyen de la direction des chutes d'eau.

En résumé, l'énergie des chutes d'eau de la Scandinavie est si considérable, et peut en général être exploitée si avantageusement, qu'elle ne tardera pas à jouer un grand rôle sur le marché de l'industrie européenne, réalisant le propos suivant que NiCola Tesla aurait tenu à un Suédois : "Pour ce qui est de votre pays, je suis d'avis qu'il va au devant d'un grand avenir. Croyez-moi, vous serez bientôt une nation riche. Auss bien l'Angleterre que l'Allemagne seraient bien aises de pouvoir dire leurs les sources infaillibles de richesses que vous possédez $)$.

SVEN LUBECK.

\section{Les Chutes d'eau de la Norvège}

Comme suite naturelle à l'article précédent, sur les chutes d'eau scandinaves, nous insérons ici la lettre suivante que nous a adressé récemment un de nos abonnés de Norvège :

Kristiania, le 24 mai 1908 .

\section{Monsieur le Rédacteur en Chef,}

J'ai lu avec le plus grand intérêt l'article inséré dans votre numéro d'avril, relatif aux forces hydrauliques en Suède et aux conditions dans lesquelles elles se présentent, et j'ai pensé qu'il y aurait peut-être intérêt pour votre Revue à être mis au courant des chutes norvégiennes. Je me permets donc de vous envoyer quelques brefs renseignements sur certaines d'entre elles, principalement sur celles qui se trouvent à l'ouest et au nosd de la Norvège.

Si l'on considère la carte hydrologique de la Norvège, on remarque que la côte ouest, qui est capricieusement découpée par des fjords profonds, s'élève rapidement à partir de la mer, et que les hauts plateaux, en deçà de ce versant, contiennent de très vastes réservoirs et d'immenses glaciers, tel le Folgefond (situé par $60^{\circ}$ de latitude nord), qui est le plus grand glacier de l'Europe.

En outre, le climat, sur la côte occidentale de la Norvège, est très caractéristique, la température moyenne y étant - sous laction du Gulfstream - assez élevée. Le tableau ci-dessous, qui indique la température moyenne, pendant les différents mois de l'année, pour trois stations distinctes de la côte, Ullensvang, Bergen et Mandal, accuse ainsi une tem pérature moyenne de 6,6 à $7,3^{\circ}$, et on verra de plus que le thermomètre, en aucun mois, n'est en moyenne descendu au-dessous de zéro. Dans la vallée de Hardanger, appelée à juste titre le jardin fruitier de la Norvège, on se livre même avec succès à la culture du tabac.

Tableau des moyennes des temptratures mensuelles

\begin{tabular}{|c|c|c|c|}
\hline Année 1903 & Ullensvang & Bergen & Mandal \\
\hline Janvier..... & $0,7^{\circ} \mathrm{C}$. & $1,33^{\circ} \mathrm{C}$. & $0,2^{\circ} \mathrm{C}$. \\
\hline Février.... & $2, I$ & 2,9 & 3,5 \\
\hline Mars & 3,8 & 4,7 & 4,3 \\
\hline Avril ..... & 4,5 & 5,1 & 4,6 \\
\hline Mai....... & 2,7 & 2,5 & 10,2 \\
\hline Juin & 13,0 & 12,3 & 14,7 \\
\hline
\end{tabular}

$\begin{array}{lccc}\text { Année r9o3 } & \text { Ullensvang } & \text { Bergen } & \text { Mandal } \\ \text { Juillet..... } & 14,1 \mathrm{C} . & 13,7 \mathrm{C} . & 14,4 \mathrm{C} . \\ \text { Août ..... } & 12,4 & 12, \mathrm{I} & 13,7 \\ \text { Septembre. } & 11,2 & 11,7 & 11,5 \\ \text { Octobre.... } & 5,6 & 7, \mathrm{l} & 6,6 \\ \text { Novembre. } & 2,5 & 3,8 & 3,3 \\ \text { Décembre.. } & 1,3 & 2,8 & 0,8 \\ \text { Moyenne.. } & 6,6^{\circ} \mathrm{C} . & 7,3^{\circ} \mathrm{C} . & 7,3^{\circ} \mathrm{C} .\end{array}$

Mais, ce qu'il y a de plus typique pour ces régions, c'est l'énorme quantité de pluie et de neige qui y tombe. Cette très grande abondance des précipitations atmosphériques s'explique tacilement par la conformation particulière du pays. En effer, les vents chargés de vapeurs, qui viennent de l'Atlantique, se trouvent subitement rencontrer, lorsqu'ils approchent de la côte, des montagnes s'élevant à une hauteur de I ooo m. et plus, et ils laissent alors tomber la plus grande partie de leur humidité sous forme de pluie, qui se concentre dans les grands bassins cités plus haut.

A titre de simple exemple, voici quelques chiffres concernant unc de ces chutes étudiée d'une façon approfondie au cours de ces dernières années, et qui représente le type caractéristique de la plupart des chutes du nord et de l'ouest de la Norvège.

Cette chute est située sur le Hardangerfjord, près d'un port toujours libre de glaces et accessible toute l'année aux plus grands navires, à 40 heures de navigation d'un port de la Manche, On peut donc y amener, de France notamment, des matériaux de tous genres qui, pour des chargements entiers, ne paieraient pas plus de 7 à 8 francs de fret par tonne. Tout autour du port s'étendent de grandes quantités de terrains propres à construcrion d'usines ou d'habitations.

La surface totale du bassin hydrologique atteint à peine $50 \mathrm{~km}^{2}$, mais il est situé à une altitude si élevée, et les précipita* tions atmosphériques y sont si intenses, qu'il fournit cependant plus de $3,2 \mathrm{~m}^{3}$ par seconde. Un barrage de $18 \mathrm{~m}$. pourrait emma* gasiner l'eau nécessaire à une bonne exploitation ininterrompue, la superficie du réservoir étant de plus de 4 kilomètres carrés.

La chute a $863 \mathrm{~m}$. de hauteur, et la longueur horizontale de la dérivation jusqu'à la mer n'est environ que de $3000 \mathrm{~m}$. La puissance hydraulique disponible toute l'année, avec exploitation de jour et de nuit, dépasserait 26.000 chevaux. L'eau serait amenée dans un tunnel de i $200 \mathrm{~m}$. percé dans la montagne et franchirait le reste de la distance dans des conduites forcées. En construisant un second barrage sur un autre petit lac, situé en amont du premier, on pourrait porter la puissance de la chute à 30000 chevaux effectifs.

Suivant des offres d'adjudication fournies par les plus impor. tantes maisons d'Europe et d'Amérique, le cheval électrique coûterait, aménagé, environ 165 francs, auxquels il faut ajouter le prix de la chute, environ 30 francs par cheval effectif, et du terrain, dont le coût ne dépasserait sans doute pas sensiblement I franc par mètre curré. Le prix total du cheval-an ne dépasserait donc guère, pour cette chute, to à 47 francs par kilowattan, et cela près d'un port de mer libre de glaces, dans un excel lent district populeux.

I'ai pensé que ces quelques notes pourraient être intéressantes pour votre estimable Revue, car je ne crois pas qu'il se trouve beaucoup d'endroits présentant des conditions plus favorables pour l'obtention de forces motrices aussi bon marché et d'un aménagement aussi facile.

Veuillez agréer, etc...

A. HIORT. 